\title{
Poroelastic response of orthotropic fractured porous media
}

\author{
James G. Berryman
}

\begin{abstract}
An algorithm is presented for inverting either laboratory or field poroelastic data for all the drained constants of an anisotropic (specifically orthotropic) fractured poroelastic system. While fractures normally weaken the system by increasing the mechanical compliance, any liquids present in these fractures are expected to increase the stiffness somewhat, thus negating to some extent the mechanical weakening influence of the fractures themselves. The analysis presented quantifies these effects and shows that the key physical variable needed to account for the pore-fluid effects is a factor of $(1-B)$, where $B$ is Skempton's second coefficient and satisfies $0 \leq B<1$. This scalar factor uniformly reduces the increase in compliance due to the presence of communicating fractures, thereby stiffening the fractured composite medium by a predictable amount. One further goal of the discussion is to determine how many of the poroelastic constants need to be known by other means in order to determine the rest from remote measurements, such as seismic wave propagation data in the field. Quantitative examples arising in the analysis show that, if the fracture aspect ratio $a_{f} \simeq 0.1$ and the pore fluid is liquid water, then for several cases considered Skempton's $B \simeq 0.9$, so the stiffening effect of the pore-liquid reduces the change in compliance due to the fractures by a factor $1-B \simeq 0.1$, in these examples. The results do however depend on the actual moduli of the unfractured elastic material, as well as on the pore-liquid bulk modulus, so these quantitative predictions are just examples, and should not be treated as universal results. Attention is also given to two previously unremarked poroelastic identities, both being useful variants of Gassmann's equations for homogeneous - but anisotropic — poroelasticity. Relationships to Skempton's analysis of saturated soils are also noted. The paper concludes with a discussion of alternative methods of analyzing and quantifying fluid-substitution behavior in poroelastic systems, especially for those systems having heterogeneous constitution.
\end{abstract}

Keywords poroelasticity $\cdot$ fractures $\cdot$ anisotropy

PACS 46.25.Cc $\cdot 46.65 .+\mathrm{g} \cdot 43.20 .+\mathrm{g} \cdot 02.50 . \mathrm{Fz}$

J. G. Berryman

Earth Sciences Division, Lawrence Berkeley National Laboratory, One Cyclotron Road MS 90R116, Berkeley, CA 94720, USA

Tel.: +510-486-5349

Fax: +510-486-5686

E-mail: jgberryman@lbl.gov 


\section{Introduction}

The most common approach used [Gassmann(1951), Skempton(1954)] when introducing poroelastic theories is to start with an implicit assumption that the unknown quantities in these systems are the fluid-saturated (or undrained) values, while the various unsaturated (or drained) values are given, or known from prior experiments. For laboratory data [Biot and Willis(1957), Berryman and Nakagawa(2010)], these assumptions may often be appropriate. But for field seismic data on oil, gas, or hydrologic reservoirs, or for sonic or ultrasonic experiments on ocean sediments, and/or for any laboratory sample where removing the pore-liquid is likely to alter the mechanical behavior of the solid matrix (or frame material) significantly due to changing surface or contact properties, these assumptions need to be carefully reexamined.

Because pertinent porous media can come in many forms, the present work is restricted to solids that might be anisotropic, up to and including those having orthotropic symmetry. Solid particles composing the porous medium frame might be arranged so the porous frame is also orthotropic in its drained constants, or the solids may be jumbled together to form a random, but still porous, polycrystal that has isotropic symmetry overall. Clearly, there are many examples of porous media left out of the mentioned classifications, including complex granular media where the grains might be either isotropic or anisotropic, homogeneous or inhomogeneous. Some of these other possibilities will be discussed elsewhere. The important additional theme to be exposed here will be pores in the form of fractures. These fractures will be assumed to be communicating (i.e., not isolated), meaning that the pore-fluid can flow from fracture to fracture, so the fracture porosity itself is connected and has finite fluid permeability. The finite permeability feature might in some circumstances be due to the original, unfractured material having had connected matrix porosity, then becoming coupled with the fractures themselves which are assumed dense enough to connect to these pre-existing, but perhaps previously unconnected porous chambers. Thus, one realization of the model could be thought of as a double-porosity (porous matrix + fracture porosity) system [Berryman and Wang(1995)]. However, we will treat only the fracture porosity itself in the present study. The matrix porosity, whenever present, is assumed to contribute to the background compliance/stiffness of the medium hosting the fractures.

The analysis that follows is intended to show how fluids affect the mechanical strength of such systems. Fractures normally weaken the system by increasing the compliance. Fluids in these fractures and other pores should be expected in most cases to increase the stiffness. We show that this is true and that the key physical variable needed to quantify this effect is the second Skempton (1954) coefficient $B$ satisfying $0 \leq B<1$, and leading to a factor $(1-B)$ that uniformly reduces the increase in compliance due to the fractures, thereby stiffening the composite medium by a predictable amount.

One additional purpose of this analysis is to determine how many of the poroelastic constants need to be known by other means in order to determine the rest from remote measurements, such as seismic wave propagation data. 


\section{Drained Bulk Modulus for Anisotropic Porous Media}

Gassmann's well-known poroelasticity equation [Gassmann(1951)] relating drained bulk modulus $K^{d}$ to undrained modulus $K^{u}$ for isotropic systems is sometimes written in the form

$$
K^{u}=K^{d}+\alpha^{2} /\left[(\alpha-\phi) / K^{g}+\phi / K_{f}\right]
$$

where $\alpha \equiv 1-K^{d} / K^{g}$ is the effective stress coefficient, or Biot-Willis coefficient [Biot and Willis(1957)], $K^{g}$ is the solid modulus of the grains (in this case assumed to be homogeneous and isotropic within all the grains), $K_{f}$ is the pore fluid modulus, and $\phi$ is porosity. The pertinent formula becomes more complicated if the solids constituting the porous medium are all isotropic but heterogeneous [Brown and Korringa(1975), Berryman and Milton(1991)], or if the grains are themselves anisotropic while not having perfectly random orientations.

The main additional complication to be treated here is one due to porosity coming in the form of fractures. The pore heterogeneity issue can also be treated, but we choose to simplify our analysis a bit for now by avoiding this additional, nontrivial extension in the present discussion. Our main goal is to show how the results for fractured systems obtained by Berryman and Grechka (2006) need to be modified when liquids are present inside these fractures.

For notational convenience, we introduce a modulus for a fluid suspension having the same solid $\left(K^{g}\right)$ and fluid $\left(K_{f}\right)$ components as well as the same porosity $\phi$ as that in the Gassmann result, but having drained modulus $K^{d} \equiv 0$. Then we find that the effective suspension modulus is given by

$$
K_{\text {susp }}=\left(\frac{1-\phi}{K^{g}}+\frac{\phi}{K_{f}}\right)^{-1} \text {. }
$$

This fact follows directly from Gassmann's formula (1) by setting $K^{d}=0$ everywhere, since then $K^{u}=K_{\text {susp }}$. But of course this result is also well-known in mechanics and acoustics for these types of fluid-solid suspensions, being the Reuss average [Reuss(1929)] and often called Wood's equation [Wood(1948)].

Rewriting Gassmann's formula in these terms, we find

$$
K^{u}=K^{d}+\frac{\left(1-K^{d} / K^{g}\right)^{2}}{1 / K_{\text {susp }}-K^{d} /\left(K^{g}\right)^{2}} .
$$

All explicit porosity $\phi$ dependence is now localized in the suspension modulus $K_{s u s p}$. From this form of the undrained equation, it is not hard to invert for the drained modulus and thereby show that it is expressible as:

$$
K^{d}=\left(\frac{K^{u}}{K_{\text {susp }}}-1\right)\left[1 / K_{\text {susp }}-2 / K^{g}+K^{u} /\left(K^{g}\right)^{2}\right] \cdot \cdot^{-1}
$$

The transition from (3) to (4) involves solution of a linear equation in $K^{d}$. So the drained modulus $K^{d}$ can be deduced easily from measurements of the undrained modulus, together with knowledge of $\phi, K_{f}$, and $K^{g}$. Apparently Zhu and McMechan (1990) first derived this simple result.

Although the result (4) as just derived applies only to isotropic and homogeneous systems, our goal will be to show that comparable results obtain for anisotropic fractured systems (up to orthotropic symmetry). Furthermore, the orthotropic poroelastic 
system of equations can also be inverted, not just for the drained (Reuss average) bulk modulus $K_{R}^{d}$, but for all the drained constants. Demonstrating these facts will be the main focus of the remainder of the paper, with special emphasis on systems having only fracture porosity (generalization to double-porosity systems will be treated elsewhere).

Consider that the overall porous medium is anisotropic due to some preferential alignment of the constituent anisotropic solid particles. We consider the orthorhombic anisotropic version of the poroelastic equations:

$$
\left(\begin{array}{c}
e_{11} \\
e_{22} \\
e_{33} \\
-\zeta
\end{array}\right)=\left(\begin{array}{cccc}
S_{11} & S_{12} & S_{13} & -\beta_{1} \\
S_{12} & S_{22} & S_{23} & -\beta_{2} \\
S_{13} & S_{23} & S_{33} & -\beta_{3} \\
-\beta_{1} & -\beta_{2} & -\beta_{3} & \gamma
\end{array}\right)\left(\begin{array}{c}
\sigma_{11} \\
\sigma_{22} \\
\sigma_{33} \\
-p_{f}
\end{array}\right)
$$

The $e_{i i}$ (no summation over repeated indices) are strains in the $i=1,2,3$ directions. The $\sigma_{i i}$ are the corresponding stresses. The fluid pressure is $p_{f}$. The increment of fluid content is $\zeta$, which (like the strains) is dimensionless. The drained compliances are $S_{i j}^{d} \equiv S_{i j}$. The drained Reuss average bulk modulus [Reuss(1929)] is defined via

$$
\frac{1}{K_{R}^{d}} \equiv \sum_{i j=1,2,3} S_{i j}^{d},
$$

a quantity which is the one commonly taken to be the definition of the bulk modulus of such a simple (non-heterogeneous) anisotropic system. Undrained compliances will be symbolized by $S_{i j}^{u}$. For the Reuss average undrained bulk modulus $K_{R}^{u}$, we have drained compliances replaced by undrained compliances in a formula otherwise identical in form to (6). Off-diagonal coefficients $\beta_{i}=S_{i 1}+S_{i 2}+S_{i 3}-1 / 3 K_{R}^{g}$, where $K_{R}^{g}$ is again the Reuss average modulus of the grains - simply replace $d$ 's with $g$ 's in (6) to determine $K_{R}^{g}$.

The alternative Voigt (1928) average [also see Hill (1952)] of the stiffnesses will play no role in this discussion. To see why this should be so, consider the Hashin-Shtrikman bounds [Hashin and Shtrikman(1963)] for the bulk moduli of composites containing isotropic constituents:

$$
\frac{1}{K_{H S}^{ \pm}+\frac{4}{3} G_{ \pm}}=\sum \frac{v_{i}}{K_{i}+\frac{4}{3} G_{ \pm}} .
$$

The upper and lower Hashin-Shtrikman bounds on bulk modulus are: $K_{H S}^{ \pm}$. Here $i=1, \ldots, n$ for the $n$ isotropic constituents. The bulk moduli of these constituents are $K_{i}$, while $G_{ \pm}$are the two most extreme values of the constituents' shear moduli: with $G_{+}$being the largest, and $G_{-}$being the smallest. Now if any of the constituents has zero shear modulus, then the lower Hashin-Shtrikman bound becomes identical to the Reuss average bulk modulus of this same system. However, the Voigt average is attainable only if one of the constituent shear moduli is infinite. While the zero shear modulus result for the lower bound produces a sensible limit - and in fact this result is exactly Wood's formula [Wood(1948)] for a suspension - the other extreme case of infinite shear modulus is surely of no interest in poroelastic systems, and perhaps never of physical interest. So we have no qualms about excluding this case from our present discussion.

Finally, coefficient $\gamma=\sum_{i=1-3} \beta_{i} / B K_{R}^{d}$ in (5), where $B$ is the second Skempton (1954) coefficient, which will be defined shortly.

The shear terms due to twisting motions (i.e., strains $e_{23}, e_{31}, e_{12}$ and stresses $\sigma_{23}$, $\left.\sigma_{31}, \sigma_{12}\right)$ are excluded from this presention because they typically do not couple to 
the modes of interest for anisotropic systems having orthotropic symmetry, or to more symmetric systems such as those having transversely isotropic or isotropic symmetry.

Summing the off-diagonal coefficients $\beta_{i}$, we find

$$
\beta_{1}+\beta_{2}+\beta_{3}=\frac{1}{K_{R}^{d}}-\frac{1}{K_{R}^{g}}=\frac{\alpha_{R}}{K_{R}^{d}}
$$

We have also introduced (similar to the isotropic case) a Reuss effective stress coefficient: $\alpha_{R} \equiv 1-K_{R}^{d} / K_{R}^{g}$. Furthermore, we have

$$
\gamma=\frac{\beta_{1}+\beta_{2}+\beta_{3}}{B}=\frac{\alpha_{R}}{K_{R}^{d}}+\phi\left(\frac{1}{K_{f}}-\frac{1}{K_{R}^{g}}\right),
$$

since a rigorous definition in this notation by Berryman (1998) for the Skempton (1954) $B$ coefficient is given by

$$
B \equiv \frac{1-K_{R}^{d} / K_{R}^{u}}{1-K_{R}^{d} / K_{R}^{g}}=\frac{\alpha_{R} / K_{R}^{d}}{\alpha_{R} / K_{R}^{d}+\phi\left(1 / K_{f}-1 / K_{R}^{g}\right)} .
$$

More general versions [Brown and Korringa(1975)] of the $B$ definition include another bulk modulus for pore response that differs from the grain response if the medium consists of a heterogeneous collection of grains and/or pores, but (as previously stated) this difference is beyond our current scope. [But see Brown and Korringa (1975) for further discussion of this point, and shall return briefly to the issue in the final technical section of the paper.] With this one caveat, all these formulas presented are rigorous statements based on anisotropic analysis. Appearances of Reuss average quantities $K_{R}^{d}$ and $\alpha_{R}$ are rigorous statements, not approximations. When grains are uniform and isotropic, there is no distinction between Voigt and Reuss averages. When grains are either anisotropic, or nonuniform in elastic properties, then it is important to note that the limiting case of Wood's formula [Wood(1948)] for a suspension of these inhomogeneous and/or anisotropic grains requires the Reuss average. Since Wood's formula is key to the definitions of both $\gamma$ and $B$, it is clear that the Reuss average is a key element of the analysis to follow. These specific choices of notation made will also help us to emphasize the similarity between rigorous anisotropic and isotropic formulas, such as (1).

\section{Off-Diagonal Poroelastic Coefficients $\boldsymbol{\beta}_{i}$}

Results for the $\beta_{i}$ coefficients are next followed by a general proof of their validity. The coefficients $\beta_{i}$ are determined by

$$
\beta_{i}=S_{i 1}^{d}+S_{i 2}^{d}+S_{i 3}^{d}-\frac{1}{3 K_{R}^{g}}
$$

where $K_{R}^{g}$ is the Reuss average of the grain modulus. Equation (11) holds for homogeneous grains, such that $K_{R}^{g}=K^{g}$. However, when the grains themselves are anisotropic, we also need to allow for this possibility by defining three directional grain bulk moduli determined by:

$$
\frac{1}{3 \bar{K}_{i}^{g}} \equiv S_{i 1}^{g}+S_{i 2}^{g}+S_{i 3}^{g}=S_{1 i}^{g}+S_{2 i}^{g}+S_{3 i}^{g},
$$


for $i=1,2,3$. The second equality follows because the compliance matrix is always symmetric. We call these quantities in (12) the partial grain-compliance sums, and the $\bar{K}_{i}^{g}$ are pertinent directional grain bulk moduli. Then, the formula in (11) is replaced by

$$
\beta_{i}=S_{i 1}^{d}+S_{i 2}^{d}+S_{i 3}^{d}-\frac{1}{3 \bar{K}_{i}^{g}}
$$

The preceding results are for perfectly aligned grains. If the grains are instead perfectly randomly oriented, then it is clear that the formulas in (11) hold as before, but now the definition of the Reuss average grain bulk modulus $K_{R}^{g}$ must be reformulated in analogy to (6).

All of these statements about the $\beta_{i}$ 's are easily proven by considering a particular combination of the applied stresses, such that $\sigma_{11}=\sigma_{22}=\sigma_{33}=-p_{c}=-p_{f}$. Then, from (5), we have:

$$
\begin{aligned}
& -e_{i i}=\left(S_{i 1}^{d}+S_{i 2}^{d}+S_{i 3}^{d}\right) p_{c}+\beta_{i}\left(-p_{f}\right) \\
& =\left(S_{i 1}^{g}+S_{i 2}^{g}+S_{i 3}^{g}\right) p_{f} \equiv \frac{p_{f}}{3 \bar{K}_{i}^{g}} \quad,
\end{aligned}
$$

in the most general of the cases discussed, and holding equally true for each value of $i=1,2,3$. This equation is a statement about the strain $e_{i i}$ (no sum over $i$ ) that would be observed in this situation, as it must be the same if these anisotropic (or inhomogeneous) grains are immersed in fluid, while measurements are taken of strains observed in each of the three directions $i=1,2,3$, during variations of this uniformly applied pressure $p_{f}$. This argument is similar to ones given by Biot and Willis (1957) for the isotropic, homogeneous case.

The relationship of coefficient $\gamma$ to the other coefficients is easily established because we have already discussed the main issue, which involves determining the role of the various other constants contained in Skempton's coefficient B. Again, from (5), we find that

$$
-\zeta=0=-\left(\beta_{1}+\beta_{2}+\beta_{3}\right) \sigma_{c}-\gamma p_{f}
$$

for undrained boundary conditions. We find that

$$
\frac{p_{f}}{p_{c}} \equiv B=\frac{\beta_{1}+\beta_{2}+\beta_{3}}{\gamma}
$$

where $p_{c}=-\sigma_{c}$ is the external confining pressure. Thus, the scalar coefficient $\gamma$ is determined uniquely, and given by

$$
\gamma=\frac{\beta_{1}+\beta_{2}+\beta_{3}}{B}=\frac{\alpha_{R} / K_{R}^{d}}{B}=\alpha_{R} / K_{R}^{d}+\phi\left(\frac{1}{K_{f}}-\frac{1}{K_{R}^{g}}\right) .
$$

This result also provides an alternative (but equivalent) definition of Skempton's second coefficient:

$$
B=\frac{\alpha_{R}}{\gamma K_{R}^{d}}
$$

although this particular result holds only for systems having homogeneous grains. 


\section{Undrained Compliance Matrix $S_{i j}^{u}$}

The undrained compliance matrix $S_{i j}^{u}$ can be found easily at this point. The general condition for undrained behavior is determined similarly to (15) by:

$$
-\zeta=0=-\left(\beta_{1} \sigma_{11}+\beta_{2} \sigma_{22}+\beta_{3} \sigma_{33}\right)-\gamma p_{f}
$$

which now relates the undrained $p_{f}$ to all the values of the applied external stresses $\sigma_{11}, \sigma_{22}$, and $\sigma_{33}$. Then, (5) is replaced by

$$
\left(\begin{array}{l}
e_{11} \\
e_{22} \\
e_{33}
\end{array}\right)=\left(\begin{array}{llll}
S_{11} & S_{12} & S_{13} & -\beta_{1} \\
S_{12} & S_{22} & S_{23} & -\beta_{2} \\
S_{13} & S_{23} & S_{33} & -\beta_{3}
\end{array}\right)\left(\begin{array}{c}
\sigma_{11} \\
\sigma_{22} \\
\sigma_{33} \\
-p_{f}
\end{array}\right)
$$

where $p_{f}=-\left(\beta_{1} \sigma_{11}+\beta_{2} \sigma_{22}+\beta_{3} \sigma_{33}\right) / \gamma$. Rewriting this expression in its simplest form gives:

$$
\left(\begin{array}{l}
e_{11} \\
e_{22} \\
e_{33}
\end{array}\right)=\left(\begin{array}{ccc}
S_{11}^{u} & S_{12}^{u} & S_{13}^{u} \\
S_{12}^{u} & S_{22}^{u} & S_{23}^{u} \\
S_{13}^{u} & S_{23}^{u} & S_{33}^{u}
\end{array}\right)\left(\begin{array}{l}
\sigma_{11} \\
\sigma_{22} \\
\sigma_{33}
\end{array}\right),
$$

where

$$
S_{i j}^{u}=S_{i j}^{d}-\frac{\beta_{i} \beta_{j}}{\gamma}
$$

To distinguish drained and undrained compliances, we have added superscripts $d$ and $u$ accordingly. Compliances without superscripts are assumed to be drained, so $S_{i j}=S_{i j}^{d}$.

\section{Role of Skempton Coefficient $B$ in Fractured Anisotropic Poroelastic Systems}

We have now determined the physical/mechanical significance of all the coefficients in the poroelastic matrix (5) from measurable quantities. The results are as general as possible $(i)$ without considering poroelastic anisotropies that have less symmetry than orthorhombic, and (ii) without allowing for mixed solids among the grains (i.e., heterogeneous composite frame). Furthermore, if the grains themselves are anisotropic, then they must be either perfectly aligned (i.e., anisotropic frame like the grains themselves) or perfectly random (i.e., isotropic polycrystalline frame). Intermediate cases of orientation including partial alignments for the case of anisotropic constituents fall into the class of anisotropic "heterogeneities," i.e., those being specifically excluded from consideration here. (Recall that our main focus in this paper is on poroelastic constants that can, at least in principle, be determined remotely, via for example laboratory ultrasound probing, or in the field using seismic experiments.)

Assumed knowledge of the three directions of the principal axes of orthotropic symmetry has also simplified this presentation somewhat. 
A remaining question is whether we can deduce all the drained constants from measured undrained ones, or not. From (5) and previous definitions including (22), the drained compliances clearly satisfy:

$$
S_{i j}^{d}=S_{i j}^{u}+\frac{B K_{R}^{d}}{\alpha_{R}} \beta_{i} \beta_{j}
$$

showing explicitly how to compute drained compliances from the measured undrained compliances. From the traditional point of view, the only unknown component on the right hand side of (23) is the undrained constants. But, for wave propagation studies, we should make the opposite assumption, i.e., we assume these undrained coefficients have been measured by carrying out undrained wave propagation experiments. So the undrained stiffnesses $C_{i j}^{u}$ are known, and presumably this $3 \times 3$ matrix has been successfully inverted for the undrained $S_{i j}^{u}$ 's. By taking appropriate sums of (23) and then using the formula (17), we find that

$$
\beta_{i} \equiv \sum_{j=1,2,3} S_{i j}^{d}-\frac{1}{3 K_{R}^{g}}=\sum_{j=1,2,3} S_{i j}^{u}-\frac{1}{3 \bar{K}_{R}^{g}}+B \beta_{i} .
$$

Rearranging (24), we obtain

$$
(1-B) \beta_{i}=\sum_{j=1,2,3} S_{i j}^{u}-\frac{1}{3 \bar{K}_{R}^{g}}
$$

where quantities on the right-hand side are assumed known. If $B$ has also been measured via the left-hand side of (16), then (23) plays the same role in the anisotropic results, as (4) did in the isotropic case.

Equation (25) is one main result, but now we can go farther and show how the same ideas could be used in the case when the porosity appears in the form of fluid-filled fractures. In this context, we make explicit use of the work of Sayers and Kachanov (1991, 1995). This approach introduces the concept of fracture-influence parameters $\eta$, measuring the increase in elastic compliance (an elastic weakening effect) due to an oriented crack in some solid elastic background material. The total effect of a set of cracks depends also on the overall crack or fracture density $\rho_{f}$, which is a pure number typically less than unity. [See Berryman and Grechka (2006) for examples of the use of numerical experiments to determine the values of a set of crack parameters $\eta$.] If the background material is the same as the grain material discussed here already, then the concept of fracture influence amounts to formulas of the form:

$$
S_{i j}^{d}=S_{i j}^{g}+\Delta S_{i j}
$$

where various corrections due to the fractures take the form $\Delta S_{i j} \propto \rho_{f} \eta$, or possibly sums of such terms. The quantity $\rho_{f}$ is the fracture density, which is related approximately to the porosity $\phi_{f}$ and the average aspect ratio $a_{f}$ of the fractures by the definition

$$
\rho_{f} \equiv \frac{\phi_{f}}{a_{f}} .
$$

See Bristow (1960), Budiansky and O'Connell (1976), and also Thomsen (2002) for careful discussions of this relationship. The main idea of these discussions is illustrated 
in Figure 1, where potential flow of a fluid past a disk (seen edge-on) is shown. The point of the illustration is to make clear that potential flow past a obstacle, whether a hard disk in a flow situation, or an empty fracture/crack in the case of elastic potential flow is strongly affected by the presence of the disk or fracture regardless of the thickness of the obstacle. Thus, the true influence does not depend on the volume of the obstacle, but rather on the volume divided by the aspect ratio. This quantity is the one computed for fractures in (27), and is one of the most useful measures of the crack or fracture density for these types of applications.

Typically we find that only two $\eta$ 's are significant at low crack densities, and we call these $\eta_{1}$ and $\eta_{2}$. For our present purposes, these details do not need to be made explicit. It will be enough to recognize that such contributions give rise to corrections of the form $\Delta S_{i j}$ as shown in (26). Then, we can show how fluids influence the effects of fractures.

If we rearrange (23) and then use (26), we have

$$
S_{i j}^{u}=S_{i j}^{d}-\frac{B K_{R}^{d}}{\alpha_{R}} \beta_{i} \beta_{j}=S_{i j}^{g}+\Delta S_{i j}-\frac{B K_{R}^{d}}{\alpha_{R}} \beta_{i} \beta_{j} .
$$

Summing this result over both $i$ and $j$, we have:

$$
\frac{1}{K_{R}^{u}}=\frac{1}{K_{R}^{g}}+\sum_{i j} \Delta S_{i j}-\frac{B \alpha_{R}}{K_{R}^{d}}
$$

since we also have

$$
\sum_{i} \beta_{i}=\sum_{i j} \Delta S_{i j}=\frac{1}{K_{R}^{d}}-\frac{1}{K_{R}^{g}}=\frac{\alpha_{R}}{K_{R}^{d}}
$$

from (26). So, we have found that

$$
\frac{1}{K_{R}^{u}}=\frac{1}{K_{R}^{g}}+(1-B) \frac{\alpha_{R}}{K_{R}^{d}}=\frac{1}{K_{R}^{g}}+(1-B)\left(\frac{1}{K_{R}^{d}}-\frac{1}{K_{R}^{g}}\right)
$$

The most important result for our present purposes then comes from combining (30) and (31) into the statement::

$$
\frac{1}{K_{R}^{u}}=\frac{1}{K_{R}^{g}}+(1-B) \sum_{i j} \Delta S_{i j}
$$

This equation shows that all the changes introduced by communicating fractures are affected in the same way, and this involves a factor of $(1-B)$ multiplying every fluidfree compliance change $\Delta S_{i j}$. The fluids therefore have the simple effect of reducing the influence of those cracks filled with fluid, since $B<1$ always holds for homogeneous poroelasticity. Thus, the general result is an overall strengthening of those parts of the material saturated with fluid by a factor of $(1-B)$. This anisotropic result is also consistent with a similar result found by the present author in isotropic porous media in an earlier publication [Berryman(2007)]. 


\subsection{Significance of the factor $(1-B)$}

To appreciate the significance of the factor $(1-B)$ which reduces the compliance of a porous medium whenever a fluid is present, first consider the fact that

$$
1-B=\frac{\phi\left(\frac{1}{K_{f}}-\frac{1}{K_{R}^{g}}\right)}{\frac{\alpha_{R}}{K_{R}^{d}}+\phi\left(\frac{1}{K_{f}}-\frac{1}{K_{R}^{g}}\right)} .
$$

Then, the term where this factor makes its most important contribution in (31) is of the form:

$$
(1-B) \frac{\alpha_{R}}{K_{R}^{d}}=\left(\frac{1}{\left(\alpha_{R} / K_{R}^{d}\right)}+\frac{1}{\phi\left(\frac{1}{K_{f}}-\frac{1}{K_{R}^{g}}\right)}\right)^{-1} .
$$

The fluid bulk modulus is assumed to lie always in the range $0 \leq K_{f} \leq K_{R}^{g}$, 1.e. from vacuum level to a value bounded above by the grain modulus of the surrounding solid materials. Then, it is straightforward to show that (34) produces a vanishing righthand side when $K_{f} \rightarrow K_{R}^{g}$, because then there is effectively no porespace left, and when $K_{f} \rightarrow 0$ we have $B \rightarrow 0$ and therefore the right hand side reduces as it should to $\alpha_{R} / K_{R}^{d}$ - which is the expected drained result. The factor $(1-B)$ therefore provides the needed interpolation factor for intermediate values of fluid $K_{f}$ in the physically pertinent range.

\subsection{Examples}

TABLE 1 displays the results of Berryman and Grechka (2006) for two models of background (both isotropic and elastic) reservoir materials: Model One has Poisson's ratio $\nu=0.00, C_{11}=C_{22}=C_{33}=13.75 \mathrm{GPa}, C_{12}=C_{13}=C_{23}=0.00 \mathrm{GPa}$, $C_{44}=C_{55}=C_{66}=G^{g}=6.875 \mathrm{GPa}$, so $K^{g}=2 G^{g} / 3=4.583 \mathrm{GPa}$, while Model Two has Poisson's ratio $\nu=0.4375$, bulk modulus $K^{g}=16.86 \mathrm{GPa}$, shear modulus $G^{g}=2.20 \mathrm{GPa}$, and $V_{p}=3.0 \mathrm{~km} / \mathrm{s}, V_{s}=1.0 \mathrm{~km} / \mathrm{s}$, with inertial mass density $\rho_{0}=2200.0 \mathrm{~kg} / \mathrm{m}^{3}$.

All these fracture-influence coefficients in TABLE 1 should be multiplied by the appropriate factor of $(1-B)$ to determine the liquid-saturated effects of these fractures on the model reservoir material.

The drained bulk modulus is obtained in this context by applying formula (18) from Berryman and Grechka (2006) :

$$
\frac{1}{K_{R}^{d}}=\frac{1}{K_{R}^{g}}+2 \rho_{f}\left[\eta_{2}+\left(\eta_{3}+\eta_{5}\right) \rho_{f}+3\left(\eta_{1}+\eta_{4} \rho_{f}\right)\right]
$$

which implies that for Model One we have

$$
\frac{\alpha_{R}}{K_{R}^{d}}=2 \rho_{f}\left(0.1941-0.2749 \rho_{f}\right) \quad \mathrm{GPa}^{-1}
$$

and for Model Two we have

$$
\frac{\alpha_{R}}{K_{R}^{d}}=2 \rho_{f}\left(0.3418-0.8250 \rho_{f}\right) \quad \mathrm{GPa}^{-1} .
$$


To compute $B$, we still need to know the values of

$$
\frac{1}{K_{s u s p}}-\frac{1}{K_{R}^{g}}=\phi_{f}\left(\frac{1}{K_{f}}-\frac{1}{K_{R}^{g}}\right) .
$$

Taking $K_{f}=2.2 \mathrm{GPa}$ for water, we find:

$$
\frac{1}{K_{\text {susp }}}-\frac{1}{K_{R}^{g}}=0.2363 \phi_{f} \quad \mathrm{GPa}^{-1}
$$

for Model One, and

$$
\frac{1}{K_{s u s p}}-\frac{1}{K_{R}^{g}}=0.3952 \phi_{f} \quad \mathrm{GPa}^{-1}
$$

for Model Two.

Formula (27) is then solved for effective fracture porosity $\phi_{f}=a_{f} \rho_{f}$. The modeling performed by Berryman and Grechka (2006) treated only the cases $\rho_{f}=0.05,0.10$, and 0.20 . Because it is the crack density $\rho_{f}$, and not the porosity itself that is important in fracture mechanics, the aspect ratio was actually not specified in that work. If the fractures are either ribbon shaped or penny-shaped, it is reasonable to assume $a_{f} \leq 0.5$, so a value of $a_{f} \simeq 0.1$ is used here in the examples found in TABLE 2 . The main observation is that $a_{f} \simeq 0.1$ results in $B \simeq 0.9$, and so the stiffening effect of the pore-liquid (water in this case) reduces the change in compliance due to the fractures by a factor $1-B \simeq 0.1$. This result does however depend on the liquid bulk modulus, as is seen in the formulas already presented.

\section{Some Further Identities in Homogeneous Poroelasticity}

One extra benefit of the preceding analysis is two identities (apparently previously unremarked) in homogeneous poroelasticity.

A related identity, but one that should be considered already known, is:

$$
\frac{1}{K_{R}^{u}}=\frac{B}{K_{R}^{g}}+\frac{1-B}{K_{R}^{d}},
$$

which is just another way of writing the well-known identity $K_{R}^{u}=K_{R}^{d} /\left(1-\alpha_{R} B\right)$, where $\alpha_{R}=1-K_{R}^{d} / K_{R}^{g}$.

But now, if we rewrite the definition of Skempton's $B$ coefficient in the form:

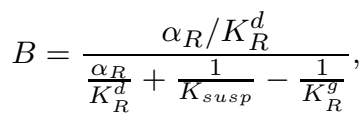

then we also find - by rearranging (42) — that

$$
\frac{1-B}{K_{R}^{d}}=\frac{1-2 B}{K_{R}^{g}}+\frac{B}{K_{\text {susp }}} .
$$

It follows that

$$
\frac{1}{K_{R}^{u}}=\frac{1-B}{K_{R}^{g}}+\frac{B}{K_{\text {susp }}}
$$


or equivalently that

$$
\frac{1}{K_{R}^{u}}-\frac{1}{K_{R}^{g}}=B\left(\frac{1}{K_{s u s p}}-\frac{1}{K_{R}^{g}}\right) .
$$

Equation (43) is another useful identity for determining the drained constant $K_{R}^{d}$, when $B$ is known. Equation (44) is obtained by substituting (43) directly into (41). Equation (45) is a rearrangement of (44).

\section{Fluid-Substitution Formulas in Homogeneous or Heterogeneous, and Anisotropic Poroelasticity}

One issue that often arises in practice for field applications of poroelasticity concerns finding means of quantifying effects related to fluid substitution (Mavko et al., 2009). The idea is simple, as the key concept comes down to being able to deduce valid relationships between and among different poroelastic data sets when the pore fluid is the only physical quantity in the poroelastic system that is changing. Examples include enhanced oil recovery (using one fluid to push another — such as oil — out of the pore spaces), geothermal systems in which a fluid (such as water or $\mathrm{CO}_{2}$ ) is injected cold into the ground and then allowed to heat up sufficiently to produce steam when extracted at a later time. Also, $\mathrm{CO}_{2}$ sequestration is another case in which the point would be to determine by remote means (such as seismic wave imaging) where the injected $\mathrm{CO}_{2}$ might be collecting underground, and residing thereafter for long periods subsequent to its injection.

One common formula used for fluid-substitution (Mavko et al., 2009) can be written in present notation as:

$$
\frac{K_{R}^{u}}{K_{R}^{g}-K_{R}^{u}}=\frac{K_{R}^{d}}{K_{R}^{g}-K_{R}^{d}}+\frac{K_{f}}{\phi\left(K_{R}^{g}-K_{f}\right)}
$$

and is based on the fact [following from (10), for example] that

$$
\frac{K_{R}^{u}}{K_{R}^{g}-K_{R}^{u}}-\frac{K_{f}}{\phi\left(K_{R}^{g}-K_{f}\right)}=\frac{1}{\alpha_{R}}-1,
$$

whenever it is appropriate to neglect the differences between pore bulk modulus $K_{\phi}$ [Brown and Korringa(1975)] and grain bulk modulus $K_{R}^{g}$ (as we have also been doing so far in this paper). The usual notation has been modified here in order to generalize the fluid-substitution statement from one that is only valid for isotropic porous media, to one that is generally valid for all anisotropic porous media in the classes considered here (up to orthotropic symmetry). We achieve this generality by replacing the isotropic bulk moduli everywhere by the corresponding Reuss average moduli of the anisotropic system (which is sufficient to guarantee consistency with Wood's formula for fluid suspensions, as discussed previously).

It is not hard to show that (47) is just one of many ways to rewrite Gassmann's equation for poroelastic systems. The significant difference in this case is that the right-hand-side of Eqn. (47) clearly does not depend at all on the pore-fluid physical properties. So, it remains constant as the pore-fluid bulk modulus is changed on the lefthand side of the equation, and also while the undrained constant $K_{R}^{u}$ is simultaneously changing in response to these fluid modulus changes. Thus, if measurements have been 
made of the system undrained response $K_{R}^{u}$ for one pore-fluid, and if the grain modulus $K_{R}^{g}$ is also known, then the right hand side of (47) has been determined. So it is then straightforward to deduce the changes in $K_{R}^{u}$ as different fluids having different $K_{f}$ 's are introduced into these same pores. Or, if the measured quantity is $K_{R}^{u}$ instead (as might be the case for seismic wave propagation studies), this result permits us to deduce the quantitative changes in pore-fluid bulk modulus $K_{f}$.

It has been shown previously [Berryman and Milton(1991)] that the most general expression of Skempton's coefficient $B$ for heterogeneous porous media is:

$$
B^{-1}=1+\frac{\phi K_{R}^{d}}{\alpha_{R}}\left(\frac{1}{K_{f}}-\frac{1}{K_{\phi}}\right),
$$

where $K_{\phi}$ is the pore bulk modulus [Brown and Korringa(1975)]. If values of $K_{\phi}$ have not been measured, then a reasonable approximation to this modulus is given by $K_{\phi} \simeq$ $K_{R}^{g}$, as previously noted. So we find the relation:

$$
B^{-1}-\frac{\phi K_{R}^{d}}{\alpha_{R} K_{f}}=1-\frac{\phi K_{R}^{d}}{\alpha_{R} K_{\phi}} \simeq 1-\frac{\phi\left(1-\alpha_{R}\right)}{\alpha_{R}},
$$

where the final step makes use of the approximation $K_{\phi} \simeq K_{R}^{d}$, as proposed after (48). Again, both right hand sides (exact and approximate, respectively) of (49) are completely independent of the pore fluid properties, as was the right hand side of (47). Equation (49) can therefore be used in the same fashion as (47) to deduce and/or predict changes in pore-fluid bulk modulus during pore-fluid injection and/or extraction processes from measured changes in $K_{R}^{u}$

\section{Conclusions}

The analysis presented here has shown that fluids in fractures increase the overall stiffness of the fractured medium. The important physical variable needed to quantify this effect is the Skempton (1954) coefficient $B$. The main result shows that a factor $(1-B)$ uniformly reduces the compliance increment caused by the presence of the fractures. The composite medium may be a solid material that is still weakened by the presence of liquid-filled fractures, but not weakened as much as it would have been if the same fractures were free of liquids. Examples based on previous work (Berryman and Grechka, 2006) show quantitatively what the magnitude of these affects can be. Results are also consistent with earlier work (Berryman, 2007), but the present approach provides a direct link between the fracture-influence parameters and Skempton's coefficient without needing to use thought experiments involving random and isotropic polycrystals of anisotropic constituents as was done in prior efforts along the same lines.

The preceding analysis has been designed to determine how many of the poroelastic constants need to be known by other means in order to determine the rest from remote measurements, such as seismic-wave propagation data. In certain cases we had to exclude some complex situations, such as anisotropic solid grains having neither perfectly random nor perfectly aligned orientations, or grains with interior pores. Clearly, other complicated circumstances may arise in practice. But to solve the corresponding poroelastic inversion problem would presumably require data such as grain-grain orientation statistics which might not be possible to obtain remotely. For laboratory measurements, the situation may be quite different however, because then it should be 


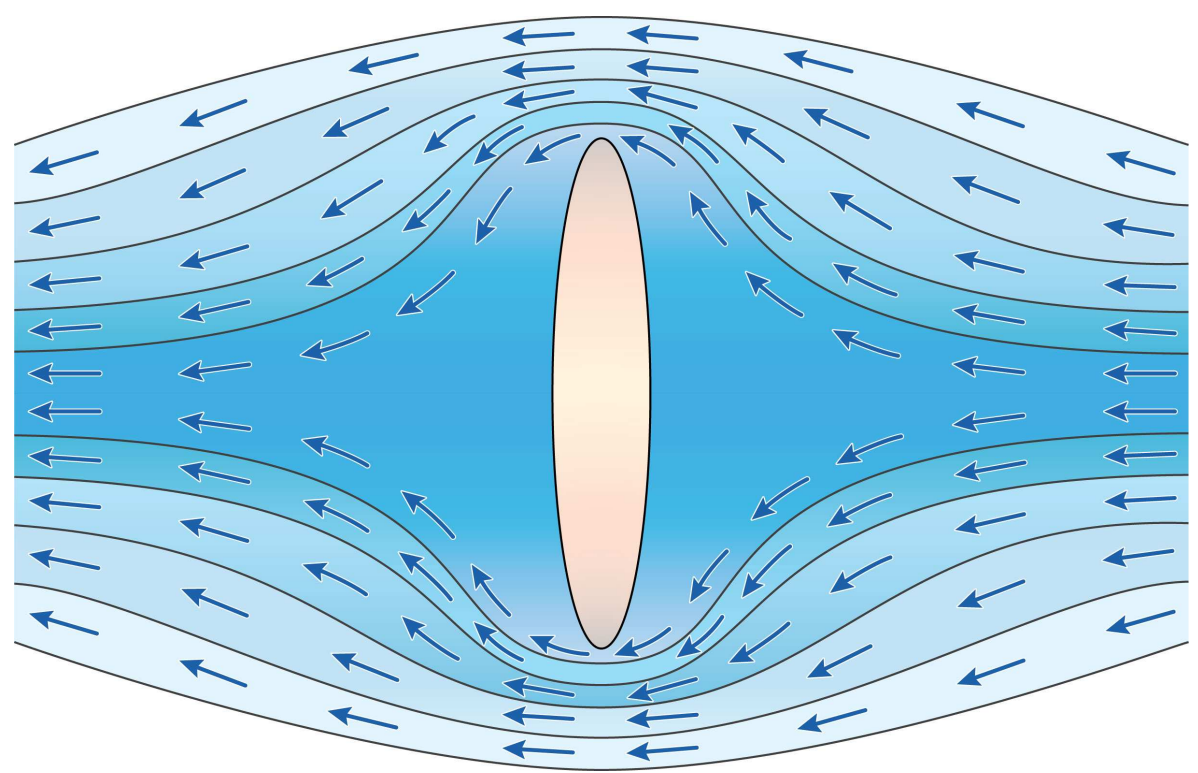

Fig. 1 Illustrating the conceptual flow analogy (based on potential flow theory) relating fracture compliance and flow around an obstacle having shape similar to that of a fracture.

Here a penny-shaped crack in an elastic material is replaced by the analogous disk-shaped obstacle (seen here edge-on) in a flow parallel to the main axis of symmetry of the disk. Flow lines change very little as the aspect ratio of the disk gets smaller, showing that the dominant physical characteristic of such flows, and elastic behavior, is controlled not by the volume of the obstacle, but rather by its volume divided by its aspect ratio.

possible to determine such grain-grain orientation parameters using x-ray tomography, or other comparable imaging tools. When such data are available, then more can (presumably) be said about both the drained and undrained poroelastic constants, as well as the relationships among them.

Finally, an alternative choice of pore-fluid substitution formula has also been derived based on the use of Skempton's coefficient $B$ in the definition of the undrained bulk modulus $K_{R}^{u}$. The main advantage of this formula is that it is completely general, and does not depend on any assumption that the porous media of interest have homogeneous (i.e., single grain-type) constitution.

Acknowledgements The author thanks Seiji Nakagawa, Mosab Nasser, and Leon Thomsen for helpful discussions of and comments on this research. Work performed under the auspices of the U.S. Department of Energy, at the Lawrence Berkeley National Laboratory under Contract No. DE-AC02-05CH11231. Support was provided specifically by the Geosciences Research Program of the DOE Office of Basic Energy Sciences, Division of Chemical Sciences, Geosciences and Biosciences. Additional support was provided by the Enhanced Geothermal Systems Demonstration Project on Induced Seismicity. All support of this research is gratefully acknowledged. 
Table 1 Values of five crack-influence parameters for the two models considered by Berryman and Grechka (2006).

\begin{tabular}{ccc}
\hline Fracture-influence & Model One & Model Two \\
Parameters $\left(\mathrm{GPa}^{-1}\right)$ & $\nu_{0}=0.00$ & $\nu_{0}=0.4375$ \\
\hline$\eta_{1}$ & 0.0000 & -0.0192 \\
$\eta_{2}$ & 0.1941 & 0.3994 \\
$\eta_{3}$ & -0.3666 & -1.3750 \\
$\eta_{4}$ & 0.0000 & 0.0000 \\
$\eta_{5}$ & 0.0917 & 0.5500 \\
\hline
\end{tabular}

Table 2 Examples of computed Skempton coefficient $B$ for the two models considered and three choices of fracture density $\rho_{f}$. Pore fluid is assumed to be water.

\begin{tabular}{ccc}
\hline $\begin{array}{c}\text { Fracture } \\
\text { Density } \\
\rho_{f}\end{array}$ & $\begin{array}{c}\text { Model One } \\
\text { Skempton } \\
B\end{array}$ & $\begin{array}{c}\text { Model Two } \\
\text { Skempton } \\
B\end{array}$ \\
\hline 0.05 & 0.9385 & 0.9447 \\
0.10 & 0.9338 & 0.9292 \\
0.20 & 0.9217 & 0.8995 \\
\hline
\end{tabular}

\section{References}

[Berryman(1998)] Berryman JG (1998) Transversely isotropic poroelasticity arising from thin isotropic layers. In: Golden KM, Grimmett GR, James RD, Milton GW, Sen PN (eds) Mathematics of Multiscale Materials, Springer-Verlag, New York, pp 37-50

[Berryman(2007)] Berryman JG (2007) Seismic waves in rocks with fluids and fractures. Geophys J Int 171:954-974

[Berryman and Grechka(2006)] Berryman JG, Grechka V (2006) Random polycrystals of grains containing cracks: Model of quasistatic elastic behavior for fractured systems. J Appl Phys 100:113,527

[Berryman and Milton(1991)] Berryman JG, Milton GW (1991) Exact results for generalized Gassmann's equations in composite porous media with two constituents. Geophysics $56: 1950-1960$

[Berryman and Nakagawa(2010)] Berryman JG, Nakagawa S (2010) Inverse problem in anisotropic poroelasticity: Drained constants from undrained ultrasound measurements. Journal of the Acoustical Society of America 127:720-729

[Berryman and Wang(1995)] Berryman JG, Wang HF (1995) The elastic coefficients of doubleporosity models for fluid transport in jointed rock. J Geophys Res 100:24,611-24,627

[Biot and Willis(1957)] Biot MA, Willis DG (1957) The elastic coefficients of the theory of consolidation. J Appl Mech 24:594-601

[Bristow(1960)] Bristow J (1960) Microcracks, and the static dynamic elastic constants of annealed and heavily cold-worked metals. Bristish Journal of Applied Physics 11:81-85

[Brown and Korringa(1975)] Brown RJS, Korringa J (1975) On the dependence of the elastic properties of a porous rock on the compressiblity of the pore fluid. Geophys 40:608-616

[Budiansky and O'Connell(1976)] Budiansky B, O'Connell RJ (1976) Elastic moduli of a cracked solid. Int J Solids Struct 12:81-97

[Gassmann(1951)] Gassmann F (1951) Über die Elastizität poröser Medien. Vierteljahrsschrift der Naturforschenden Gesellschaft in Zürich 96:1-23

[Hashin and Shtrikman(1963)] Hashin Z, Shtrikman S (1963) A variational approach to the theory of the elastic behavior of multiphase materials. J Mech Phys Solids 11:127-140

[Hill(1952)] Hill R (1952) Elastic properties of reinforced solids: Some theoretical principles. Proc Phys Soc London A 65:349-354

[Mavko et al(2009)Mavko, Mukerji, and Dvorkin] Mavko G, Mukerji T, Dvorkin J (2009) The Rock Physics Handbook: Tools for Seismic Analysis of Porous Media (Second Edition). Cambridge University Press, Cambridge, England 
[Reuss(1929)] Reuss A (1929) Berechung der Fließgrenze von Mischkristallen auf Grund der Plastizitätsbedingung für Einkristalle. Z Angew Math Mech 9:49-58

[Sayers and Kachanov(1991)] Sayers CM, Kachanov M (1991) A simple technique for finding effective elastic constants of cracked solids for arbitrary crack orientation statistics. Int J Solids Struct 27:671-680

[Sayers and Kachanov(1995)] Sayers CM, Kachanov M (1995) Microcrack-induced elastic wave anisotropy of brittle rocks. J Geophys Res 100:4149-4156

[Skempton(1954)] Skempton AW (1954) Coherent-potential model of substitutional alloys. Géotechnique 4:143-147

[Thomsen(2002)] Thomsen L (2002) Understanding Seismic Anisotropy in Exploration and Exploitation. Society of Exploration Geophysicists, Tulsa, OK

[Voigt(1928)] Voigt W (1928) Lehrbuch der Kristallphysik. Teubner, Leipzig

[Wood(1948)] Wood AW (1948) A Textbook of Sound. Bell, London

[Zhu and McMechan(1990)] Zhu X, McMechan GA (1990) Direct estimation of the bulk modulus of the frame in a fluid-saturated elastic medium by Biot theory. In: 60th International Meeting, Society of Exploration Geophysicists, p 787 


\section{DISCLAIMER}

This document was prepared as an account of work sponsored by the United States Government. While this document is believed to contain correct information, neither the United States Government nor any agency thereof, nor The Regents of the University of California, nor any of their employees, makes any warranty, express or implied, or assumes any legal responsibility for the accuracy, completeness, or usefulness of any information, apparatus, product, or process disclosed, or represents that its use would not infringe privately owned rights. Reference herein to any specific commercial product, process, or service by its trade name, trademark, manufacturer, or otherwise, does not necessarily constitute or imply its endorsement, recommendation, or favoring by the United States Government or any agency thereof, or The Regents of the University of California. The views and opinions of authors expressed herein do not necessarily state or reflect those of the United States Government or any agency thereof or The Regents of the University of California.

Ernest Orlando Lawrence Berkeley National Laboratory is an equal opportunity employer. 